\title{
Manejo nutricional del paciente con úlceras por presión: una revisión de la literatura
}

\author{
Nutritional management of pressure ulcers: A review of literature \\ Manejo nutricional do paciente com úlcera por pressão: uma revisão da literatura \\ Claudia Patricia Maza Moscoso ${ }^{1,2}$, Iván Armando Osuna-Padilla ${ }^{3 *}$, Patricia Paulina Maldonado Valadez ${ }^{4}$
}

Recibido: 22 de junio de 2020. Aceptado para publicación: 7 de septiembre de 2020

Publicado en línea: 10 de septiembre de 2020

https://doi.org/10.35454/rncm.v4n1.162

\begin{abstract}
Resumen
Las heridas crónicas se presentan con frecuencia en adultos hospitalizados y personas de la tercera edad institucionalizadas. Su desarrollo tiene una relación importante con el estado nutricional. El objetivo de esta revisión narrativa es analizar la relación entre la nutrición y la incidencia de úlceras por presión, además de estudiar las estrategias de abordaje nutricional sugeridas por la evidencia científica, haciendo énfasis en el aporte de energía, proteínas, aminoácidos y otros micronutrientes. La nutrición es un factor influyente en la cicatrización de heridas; sin embargo, se necesitan más estudios para establecer los aportes adecuados de ciertos nutrientes y el impacto que tendrán en la evolución de esta condición.
\end{abstract}

Palabras clave: estado nutricional, nutrientes.

\begin{abstract}
Chronic wounds occur frequently in adult hospitalized patients and institutionalized seniors. Their occurrence has a close relationship with nutritional status. The objective of this narrative review is to analyze the relationship between nutrition and the incidence of pressure ulcers, as well as to study the nutritional strategies recommended in scientific literature, focusing on the contribution of energy, proteins, amino acids and other micronutrients. Nutrition is an influential factor in wound healing. However, additional studies are needed to establish the adequate intake of certain nutrients, and the impact these have on the progression of this condition.
\end{abstract}

Keywords: Nutritional status; Nutrients.

\section{Resumo}

As feridas crônicas ocorrem com frequência em adultos hospitalizados e idosos institucionalizados. O seu desenvolvimento, tem importante relação com o estado nutricional. O objetivo desta revisão narrativa é analisar a relação entre a nutrição e a incidência de úlceras por pressão, além de estudar as estratégias de abordagem nutricional sugeridas pela evidência científica, enfatizando o contributo do fornecimento de energia, proteínas, aminoácidos e outros micronutrientes. A nutrição é um fator influente na cicatrização de feridas; porem, é necessários mais estudo para estabelecer as contribuições adequadas de certos nutrientes e o impacto que eles terão na evolução desta condição.

Palavras-chave: estado nutricional, nutrientes.
1 Departamento de Dietética y Alimentación. Centro Médico Militar. Ciudad Guatemala. Guatemala.

2 Universidad del Valle de Guatemala. Ciudad de Guatemala. Guatemala

*Correspondencia: Iván A. Osuna- Padilla

ivan.osuna@cieni.org.mx
3 Centro de Investigación en Enfermedades Infecciosas, Instituto Nacional de Enfermedades Respiratorias, Ciudad de México, México.

4 Departamento de Nutrición, Hospital General de León, León, Guanajuato.

\section{INTRODUCCIÓN}

Las úlceras por presión (UPP) son un problema común que suelen desarrollar muchos pacientes hospitaliza- dos, en especial adultos mayores. Son definidas como la presencia de necrosis isquémica al nivel de la piel y los tejidos subcutáneos, la cual se produce generalmente por la presión ejercida entre dos planos duros, 
que provoca una disminución del aporte de oxígeno y nutrientes a esa zona ${ }^{(1)}$.

Las UPP se clasifican en cuatro estadios dependiendo del grado de afectación. Estadio I) presencia de eritema cutáneo que no palidece. Estadio II) presencia de pérdida parcial del grosor de la piel, pudiéndose ver afectada la epidermis y la dermis. Estadio III) pérdida total del grosor de la piel, con presencia de lesión o necrosis del tejido subcutáneo, cuya profundidad no afecta la fascia subyacente. Estadio IV) ocurre afectación de todo el grosor de la piel, observándose necrosis tisular o daño en el músculo o hueso ${ }^{(1,2)}$.

La prevalencia documentada es alta, reportándose que $1,1 \%$ a $26 \%$ de pacientes hospitalizados, $6 \%$ a $29 \%$ de pacientes ambulatorios, 7,6 \% a 53,2\% de residentes de asilos y $13,1 \%$ a $28,7 \%$ de pacientes en la unidad de cuidados intensivos suelen desarrollarlas ${ }^{(2)}$. La presencia de UPP se asocia a mayor riesgo de muerte, siendo mayor el riesgo en aquellos con infección en la zona y neutrofilia ${ }^{(3)}$. Se estima que 60.000 pacientes mueren por complicaciones en los Estados Unidos, siendo las UPP un factor de riesgo para ello, además de incrementar la estancia hospitalaria en 4,31 días en comparación con adultos que no las desarrollaron ${ }^{(4,5)}$.

El proceso de cicatrización incluye fases como la hemostasis, inflamación, proliferación y remodelación. Después de la injuria, la fase de la hemostasis es desencadenada y se acompaña por la contracción vascular, agregación plaquetaria y formación de fibrina, dando paso a la fase inflamatoria, donde plaquetas, macrófagos y células de la médula ósea segregan factores que promueven la activación de los fibroblastos en el sitio dañado e inician la fase proliferativa. Los fibroblastos al secretar colágeno, glicosaminoglicano y proteoglicanos, inician la fase de remodelación de la herida. Esta fase es un proceso largo, que puede durar meses o años, dependiendo de las características de la herida y comorbilidades del paciente ${ }^{(6,7)}$. Las heridas crónicas se definen como aquellas que no cicatrizan de manera normal y no tienen una efectiva cicatrización en tres meses, y se caracterizan según el proceso por falla en completar las fases, no logrando avanzar de la fase inflamatoria ${ }^{(8)}$.

La relación entre la nutrición y la cicatrización ha sido reconocida por décadas, describiéndose que para llevarse a cabo una correcta cicatrización se requiere un consumo adecuado de energía, proteína y micronutrientes ${ }^{(9)}$. La desnutrición ha mostrado ser un determinante del desarrollo de UPP, documentándose que el paciente con desnutrición tiene $129 \%$ mayor riesgo de desarrollarlas (RR 2,29, IC95 \% 1,53-3,44), indepen- dientemente de otros factores predictores como edad $y$ comorbilidades, entre otros ${ }^{(10)}$. Además, existe una relación significativa entre pérdida de peso involuntaria, un IMC $<18,5 \mathrm{~kg} / \mathrm{m}^{2}$ y la baja ingesta nutricional con la incidencia de $\mathrm{UPP}^{(5)}$. Un estudio realizado por Banks y colaboradores logró mostrar que la intervención nutricional es un enfoque de utilidad en la prevención de la UPP, al demostrar el ahorro de 2.869,526 euros (SD 2078 715) cuando los pacientes recibieron una intervención nutricional, en comparación con la atención estándar ${ }^{(11)}$. Es por esto que la desnutrición debe identificarse de forma oportuna para definir las directrices dietéticas que permitan una cicatrización adecuada ${ }^{(12)}$. Además de una alimentación e hidratación adecuada, se han estudiado otras intervenciones que pueden tener beneficios significativos en la cicatrización de heridas, sugiriéndose la suplementación de arginina, glutamina y micronutrientes ${ }^{(13)}$.

El objetivo de esta revisión narrativa es analizar los factores nutricionales que influyen en la incidencia de UPP. Se analizan las estrategias de abordaje nutricional sugeridas por la evidencia científica, haciendo énfasis en el aporte de energía, proteínas, aminoácidos y otros micronutrientes. Además, se propone un algoritmo de manejo nutricional en el manejo de las UPP.

\section{FACTORES DE RIESGO PARA EL DESARROLLO DE UPP}

El proceso de cicatrización está influenciado por diversos factores no modificables, como la edad, el esquema de medicamentos, las concentraciones de hormonas sexuales, la presencia de infecciones y diabetes, entre otros. Otros factores de riesgo modificables han cobrado relevancia, entre los que destacan el estrés psicológico, tabaquismo, alcohol y el consumo subóptimo de nutrientes ${ }^{(14)}$.

\section{Consumo subóptimo de nutrientes}

Un consumo adecuado de nutrientes resulta extremadamente importante para la regulación de todas las fases que influyen en la cicatrización de heridas, así como la capacidad del cuerpo para poder favorecer la cicatrización de una manera adecuada. Dichos nutrientes son necesarios para la síntesis de ácidos nucleicos (ARN y ADN), proteínas y otros factores involucrados en la maduración y diferenciación tisular. El consumo óptimo de hidratos de carbono es importante para proveer la energía para la angiogénesis y deposición de nuevos tejidos. El consumo subóptimo de proteí- 
nas altera la formación de capilares, la proliferación de fibroblastos, la síntesis de proteoglucanos y de colágeno, afectando con ello la cicatrización, mientras que distintas vitaminas como la A, C, E y minerales como el magnesio, zinc y el hierro, tienen un rol importante como cofactores de distintas enzimas involucradas en la síntesis proteica y de colágeno ${ }^{(14,15)}$.

\section{Malnutrición}

La proliferación celular y síntesis proteica durante el proceso de cicatrización hace que el cuerpo aumente sus necesidades nutricionales, siendo la desnutrición una causa y también una consecuencia de la presencia de $\mathrm{UPP}^{(9)}$. La prevalencia de las UPP es mayor en pacientes con desnutrición, según lo documentado por Shanin et al., al observar mayor incidencia en pacientes hospitalizados que cursaron con pérdida de peso, IMC $<18,5 \mathrm{~kg} /$ $\mathrm{m}^{2}$ o disminución en el consumo de alimentos ${ }^{(5)}$. Fry et al., también reportaron que la malnutrición preexistente y pérdida de peso son factores predictivos de condiciones quirúrgicas no deseadas y adquiridas en el ámbito hospitalario, incluyendo $\mathrm{UPP}^{(16)}$. La asociación entre desnutrición y UPP radica en que la primera ocasiona alteraciones en la integridad de la piel (adelgazamiento de la piel), inmunosupresión, disminución en la proliferación de fibroblastos y en la síntesis de colágeno provocando con ello retrasos en la cicatrización, incrementando con ello el riesgo de UPP e infección de las mismas ${ }^{(17,18)}$.

\section{Obesidad}

Las personas con obesidad están propensas a padecer varias patologías severas, incluyendo dificultad en la cicatrización, la cual puede ser explicada por hipoperfusión y efectos isquémicos que ocurren en el tejido adiposo subcutáneo ${ }^{(19)}$. Esto impide la infiltración de células inmunes en el área de la herida, a lo que se suma dehiscencia de la misma por el aumento de la tensión de las orillas de la herida y por la invasión de bacterias residentes ${ }^{(20)}$. En un estudio transversal, Ness et al., documentaron que los pacientes con IMC $>35 \mathrm{~kg} / \mathrm{m}^{2}$, presentan tres veces más riesgo de desarrollar las UPP en comparación con individuos con un IMC dentro del rango de normalidad (RR 3,478, IC95 \% 1,65 - 7,3, p = 0,001) ${ }^{(21)}$.

\section{Diabetes y enfermedades cardiovasculares}

La diabetes mellitus ocasiona diversas alteraciones en los procesos y fases de la cicatrización. Las personas con diabetes muestran una deficiente quimiotaxis de los neutrófilos, así como en las actividades fagocíticas y microbicidas, incrementando el riesgo a infecciones. La infiltración celular, activación insuficiente de macrófagos, disminución en la secreción de factor de necrosis tumoral alfa (TNF- $\alpha$ ) e interleucina 1 (IL-1 $\beta$ ) y disminución en la funcionalidad de leucocitos han demostrado un impacto negativo en la cicatrización de heridas ${ }^{(14,22)}$.

El riesgo de infecciones en heridas (RR 2,7, IC95 \% $1,59-4,62, \mathrm{p}<0,001)$ y dehiscencia de las mismas (RR 4,09, IC95 \% 2,49 - 6,74, p< 0,001) es mayor en el paciente quirúrgico con diagnóstico de diabetes ${ }^{(23)}$, debido a que la inflamación sistémica ocasiona retraso en la deposición de los componentes de la matriz, remodelado y cicatrización de la herida ${ }^{(24)}$.

La presencia de alteraciones cardiovasculares, como ateroesclerosis, falla cardiaca congestiva, enfermedad cardiovascular periférica y eventos cerebrovasculares, incrementan el riesgo a desarrollar UPP, ya que la disminución en el gasto cardiaco condiciona hipotensión, menor perfusión sanguínea e isquemia periférica ${ }^{(18,25,26)}$. Un estudio retrospectivo de cerca de 50.000 pacientes mostró una incidencia de UPP durante la estancia hospitalaria de $33,9 \%$ en pacientes que habían sufrido un evento cerebrovascular y de $43,8 \%$ en pacientes con falla cardiaca congestiva ${ }^{(27)}$.

\section{INTERVENCIÓN NUTRICIONAL PARA EL MANEJO Y PREVENCIÓN DE UPP}

Las guías de la European Pressure Ulcer Advisory Panel (EPUAP) para la prevención y tratamiento de UPP, publicadas en el 2019, consideran esencial la terapia nutricional, emitiendo distintas recomendaciones para la prescripción dietética. En el paciente hospitalizado se debe promover el consumo de alimentos vía oral antes de la indicación de otras estrategias, por lo que se sugiere la prescripción de la dieta hospitalaria de forma individualizada, considerando modificaciones en consistencia en casos donde así se amerite, incorporando alimentos fortificados, ofreciendo asistencia para el consumo de alimentos y un ambiente tranquilo al momento del consumo, así como la evaluación de la ingesta de la dieta prescrita ${ }^{(28)}$.

\section{Energía}

En pacientes con UPP se debe dar un aporte adecuado de energía y una alimentación equilibrada en macronutrientes para que la proteína sea utilizada en la formación de la estructura celular y síntesis de colágeno y no en las vías energéticas. Heridas pequeñas tienen poco impacto 
en el gasto energético, pero heridas complicadas, pueden necesitar un alto aporte de energía para la cicatrización. La medición del requerimiento de energía debe hacerse idealmente por calorimetría indirecta, sin embargo, esta técnica es poco accesible en los centros hospitalarios. En ausencia de la misma, se sugiere la prescripción de 30 $35 \mathrm{kcal} / \mathrm{kg}$, utilizando el juicio clínico para la elección del peso a utilizar (por ejemplo, peso ideal en caso de desnutrición, peso ajustado en obesidad y peso seco en condiciones de sobrehidratación) para evitar condiciones de sub y sobrealimentación ${ }^{(28,29)}$.

Los hidratos de carbono y los lípidos son las fuentes primarias de energía en el cuerpo, jugando un papel importante en el proceso de cicatrización, sin embargo, no existen recomendaciones de las cantidades óptimas a prescribir, por lo que considerando lo estipulado para una alimentación saludable, se sugiere de $45 \%$ - $65 \%$ de hidratos de carbono y de $20 \%$ - $35 \%$ de lípidos ${ }^{(30)}$. Poco se ha explorado respecto a las fuentes alimentarias de estos nutrientes, documentándose que la glicación avanzada asociada al consumo de hidratos de carbono no saludables (azúcares simples, productos industrializados) afecta la fase de granulación al disminuir el recambio de colágeno tipo I, mientras que los ácidos grasos omega 3 pueden ser de utilidad al promover la inmunomodulación del hospedero más que la cicatrización per se, al poseer propiedades antiinflamatorias que inhiben la producción de eicosanoides y otros mediadores como el factor de activación plaquetario, IL-1 y TNF- $\alpha^{(31)}$.

\section{Proteínas}

Las proteínas son esenciales en todas las etapas de la cicatrización de las UPP. Participan en la multiplicación celular, síntesis de colágeno y tejido conectivo, así como también para la preservación de la función inmune, reducción del tamaño y profundidad de las UPP. Por lo tanto, el aporte debe de ser adaptado a las necesidades de cada individuo dependiendo del número y severidad de úlceras por presión, estado nutricional, comorbilidades y tolerancia a la alimentación ${ }^{(17)}$, recomendándose compensar las pérdidas de nitrógeno en el exudado de la UPP ${ }^{(26)}$. Los resultados de un ensayo clínico publicado por Ohura et al., en 2011 mostraron mejorías en cicatrización tras 8 semanas de una intervención nutricional alta en calorías y proteína $(37,9 \mathrm{kcal}$ y $1,62 \mathrm{~g} / \mathrm{kg})$ en comparación con una intervención estándar $(29,1 \mathrm{kcal}$ y $1,24 \mathrm{~g} / \mathrm{kg})^{(32)}$, por lo que las guías EPUAP sugieren el aporte proteico en un rango de 1,25 a 1,5 g/ kg/ día ${ }^{(28)}$.
En los últimos años se ha descrito que la utilidad del balance nitrogenado puede causar controversia en pacientes con fístulas, heridas, quemaduras y úlceras, al subestimar las pérdidas de nitrógeno a través de fluidos distintos a la orina, además de requerir una cuantificación exacta del nitrógeno ingerido por el paciente, lo que se vuelve complicado en el paciente con alimentación por vía oral ${ }^{(33)}$. Sin embargo, en este contexto, se debe valorar el someter a estos pacientes a un balance nitrogenado de control, interpretando con cautela los resultados para estimar un adecuado aporte proteico.

\section{Hidratación}

El agua está distribuida en el cuerpo y es un medio de transporte de nutrientes y desechos, por lo que el equipo interdisciplinario debe monitorizar el estado de hidratación de los pacientes y promover el consumo óptimo de líquidos, los cuales dependerán de la condición clínica de cada individuo, requiriendo mayores cantidades quienes cursan con fiebre, vómito, sudoración, diarrea o heridas que drenan líquido, sugiriéndose el aporte de $1 \mathrm{~mL}$ de agua por cada caloría consumida ${ }^{(28)}$.

\section{SUPLEMENTACIÓN DE NUTRIENTES}

Adicional a un correcto aporte de energía y proteínas, se ha estudiado el impacto de diferentes estrategias de suplementación de aminoácidos, vitaminas y elementos traza ${ }^{(15)}$.

\section{Aminoácidos}

Los aminoácidos como la arginina y glutamina son condicionalmente esenciales en períodos de estrés severo como trauma y UPP. La arginina es sintetizada de la ornitina a través de la citrulina. Este aminoácido juega un papel esencial en la cicatrización a través de distintos mecanismos: 1) al ser un precursor de la prolina en la síntesis de colágeno, 2) al promover la secreción del factor de crecimiento de la insulina, 3) al actuar como un estimulador de linfocitos $\mathrm{T}$ y 4 ) al ser un sustrato que genera óxido nítrico ${ }^{(13,34)}$. Los ensayos clínicos que evalúan la efectividad de la suplementación de arginina son limitados. Desneves et al., observaron mejorías en cicatrización tras suplementar durante 3 semanas un suplemento oral nutricional alto en energía y proteína enriquecido con arginina $(9 \mathrm{~g})$, vitamina C (500 mg) y zinc $(30 \mathrm{mg})^{(35)}$. Resultados similares fueron reportados por van Anholt et al., quienes prescribieron un suplemento oral nutricional $(200 \mathrm{~mL}, 250 \mathrm{kcal}, 20 \mathrm{~g}$ 
de proteínas, $3 \mathrm{~g}$ de arginina) durante 8 semanas ${ }^{(36)}$. La metodología utilizada en ambos estudios no permite elucidar el rol de la arginina en la cicatrización, debido a que la suplementación se ha realizado conjunto con proteínas y otros micronutrientes que también influyen en dicho proceso.

La glutamina es el aminoácido más abundante en el cuerpo. Este aminoácido es un importante precursor de la síntesis de nucléotidos en las células, incluyendo fibroblastos y macrófagos, además de tener un rol esencial en la proliferación de linfocitos ${ }^{(37)}$. Gran parte de la evidencia disponible proviene de estudios in vitro o modelos animales. Blass et al., observaron mejorías en la cicatrización al prescribir durante 14 días un suplemento nutricional con glutamina (20 g), ácido ascórbico $(500 \mathrm{mg})$ y zinc $(6,6 \mathrm{mg})$, documentando menor número de días transcurridos hasta el cierre de la herida $(35 \pm 22)$ en el grupo con suplementado en comparación con un grupo placebo $(70 \pm 35 \text { días })^{(38)}$.

\section{Vitaminas}

La vitamina A es requerida para el desarrollo y mantenimiento de la integridad del tejido epitelial y una correcta cicatrización, así como regular genes que codifican proteínas estructurales, por lo que se sugiere cubrir la ingesta diaria recomendada (IDR) para población sana (900 $\mu \mathrm{g}$ en hombres y $700 \mu \mathrm{g}$ en mujeres) a través de fuentes alimentarias. Existe poca evidencia proveniente de estudios en humanos que respalde la suplementación a dosis supra fisiológicas, por lo que no existe consenso de las dosis a suplementar, sugiriéndose dosis entre $3.000-15.000 \mu \mathrm{g}$ de retinol ${ }^{(16)}$.

En el paciente con UPP, el consumo óptimo de vitamina C (90 mg/día en hombres y $75 \mathrm{mg} /$ día en mujeres) ${ }^{(28)}$ es importante, ya que promueve la formación de colágeno y estimula la activación de leucocitos y macrófagos ${ }^{(39)}$. Algunos autores sugieren la suplementación a dosis superiores $(250-2.000 \mathrm{mg} /$ día $)$; $\sin$ embargo, no existe evidencia científica que respalde esta recomendación ${ }^{(17)}$. Un estudio publicado por Riet et al., no observó mejorías en la cicatrización tras suplementar $1.000 \mathrm{mg} /$ día de ácido ascórbico en pacientes con $\mathrm{UPP}^{(40)}$.

La vitamina E mantiene y estabiliza la integridad de la membrana celular, protegiéndola de daño por especies reactivas de oxígeno, además de modular la expresión del factor de crecimiento de tejido conectivo ${ }^{(28)}$. No existe consenso de las dosis adecuadas a suplemen- tar, por lo que se debe promover el consumo óptimo a través de la vía oral.

\section{Micronutrientes}

Diversos micronutrientes juegan un rol importante en la cicatrización al ser cofactores de enzimas ${ }^{(13)}$.

El magnesio es esencial en la reparación de heridas $y$ funciona como cofactor de muchas enzimas que ayudan a la síntesis de proteína y colágeno. Proporciona estabilidad estructural al ATP, que ayuda a muchos de los procesos utilizados en la síntesis de colágeno. El hierro es necesario para el transporte de oxígeno y la hidroxilación de la prolina y lisina, existiendo alteraciones en la producción de colágeno en condiciones de deficiencia. El cobre es un cofactor en el metabolismo del colágeno, viéndose afectada la cicatrización en pacientes con depleción de las reservas corporales ${ }^{(13,17,28)}$. No existen recomendaciones de dosis para dichos micronutrientes.

El zinc es un cofactor para la formación de colágeno y juega un papel importante en la síntesis de proteína. Es transportado en el cuerpo por la albúmina y su absorción disminuye cuando la albúmina disminuye en situaciones como trauma, sepsis o infección. Su deficiencia puede resultar de un aumento en el drenaje de las heridas, disminución de la ingesta dietética y aumento de las pérdidas gastrointestinales, teniendo por consecuencia pérdida de apetito, sentido del gusto alterado, alteración de la función inmune y problemas para cicatrización de heridas ${ }^{(41-43)}$. Las guías EPUAP sugieren no suplementar este nutriente de forma rutinaria ${ }^{(28)}$, sin embargo, un metaanálisis publicado por Song et al., mostró mejorías en cicatrización tras evaluar los resultados de siete ensayos clínicos (RR 1,44, IC95 \% $1,01-2,06, p=0,043)$, los cuales utilizaban diferentes presentaciones de zinc (crema, aceite, suplemento oral, suplemento oral nutricional) ${ }^{(44)}$. La suplementación con zinc puede acelerar la cicatrización, pero se necesitan más estudios que esclarezcan las dosis y seguridad de la suplementación.

Si hay signos clínicos de una deficiencia, debe ser suplementado en una cantidad no mayor de $40 \mathrm{mg}$ de zinc elemental al día. Las dosis altas no son recomendadas porque pueden afectar de manera adversa el estatus del cobre y resultar en anemia. Los altos niveles de zinc pueden inhibir la cicatrización, afectar la fagocitosis e inducir a deficiencia de cobre, ya que compiten por los receptores en la molécula de albúmina ${ }^{(41)}$. Antes 
de recomendar la suplementación, se debe evaluar el consumo a través de la alimentación, además de las cantidades aportadas por productos fortificados como fórmulas enterales. En la Figura 1 se menciona el rol de los nutrientes en el proceso de cicatrización.

\section{Suplementos nutricionales}

De acuerdo con un metaanálisis publicado en 2014, existe evidencia insuficiente que respalde la efectividad de los suplementos orales nutricionales en la prevención o tratamiento de las UPP. Para esta publicación se analizaron los datos provenientes de 11 ensayos clínicos aleatorizados que utilizaban diferentes estrategias de suplementación ${ }^{(47)}$. Otro metaanálisis publicado por Cereda et al., documentó mejorías en la cicatrización tras evaluar tres ensayos clínicos de suplementación de fórmulas que contenían arginina, zinc y antioxidantes ${ }^{(46)}$, documentándose en un análisis de costo efectividad a largo plazo, que el uso de suplementos orales nutricionales conlleva menos costo que el tratamiento de las $\mathrm{UPP}^{(47,48)}$. Considerando esto, las guías EPUAP sugieren la suplementación oral nutricional con productos altos en energía y proteína en aquellos individuos que no logran cubrir las necesidades nutricionales a través de la vía oral. La recomendación es brindar terapia nutricional mediante la administración de productos hiperproteicos y energéticamente densos con una duración mínima de 1 mes o hasta que la herida cicatrice ${ }^{(28)}$.

La Figura 2 presenta un algoritmo del abordaje nutricional del adulto hospitalizado con UPP. Este algoritmo se fundamenta en la literatura aquí resumida, que incluyen las recomendaciones de manejo de EPUAP.

\section{Soporte nutricional especializado}

De manera general, se recomienda el uso de nutrición enteral en aquellos pacientes que no alcanzan a cubrir su requerimiento vía oral o por alguna condición no puede ingerir alimentos vía oral y requiere la colocación de alguna sonda para alimentación, siempre y cuando $\mathrm{su}$ tracto gastrointestinal se encuentre funcional. El uso de nutrición parenteral se sugiere en los pacientes que tengan el tracto gastrointestinal disfuncional o que éste amerite reposo por lo que se encuentran en ayuno franco, o cuando los requerimientos de vía oral o enteral no son cubiertos ${ }^{(26)}$.

\section{CONCLUSIONES}

Todo paciente con UPP debe ser evaluado nutricionalmente y tener un plan de cuidado nutricional individualizado. El propósito del plan de cuidado nutricional es mantener o mejorar el estado nutricional y la función inmunológica, promoviendo con ello una adecuada cicatrización. Los hallazgos de la evaluación nutricional exhaustiva marcarán la pauta para definir las estrategias terapéuticas más adecuadas, como la incorporación de suplementos orales, y estrategias de suplementación de micronutrientes, entre otros. A pesar de que una gran cantidad de estudios han evaluado la efectividad de distintas estrategias en la cicatrización de UPP, existe poco consenso debido a las diferencias metodológicas empleadas, no existiendo lineamientos respecto a cuánto y durante cuánto tiempo suplementar. Considerando esto, los esfuerzos deben enfocarse en lograr el consumo vía oral de las cantidades adecuadas

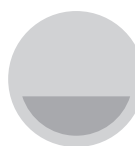

\section{Fase inflamatoria}

Vitamina A. Promueve la

fase inflamatoria temprana

Proteínas. Previenen la

prolongación de la fase

inflamatoria

Vitamina C. Promueve la

migración de macrófagos

y la funcionalidad de

linfocitos

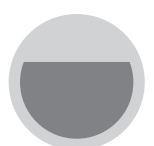

Fase proliferativa

Vitamina C. Necesaria para

la síntesis de colágeno

Vitamina A. Promueve la

diferenciación de células

epiteliales

Zinc. Necesario para la síntesis de ADN, división

celular y síntesis proteica

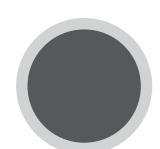

Fase de remodelación

Aminoácidos y proteínas.

Esenciales para el

remodelado de heridas

Figura 1. Rol de la nutrición en el proceso de cicatrización. 


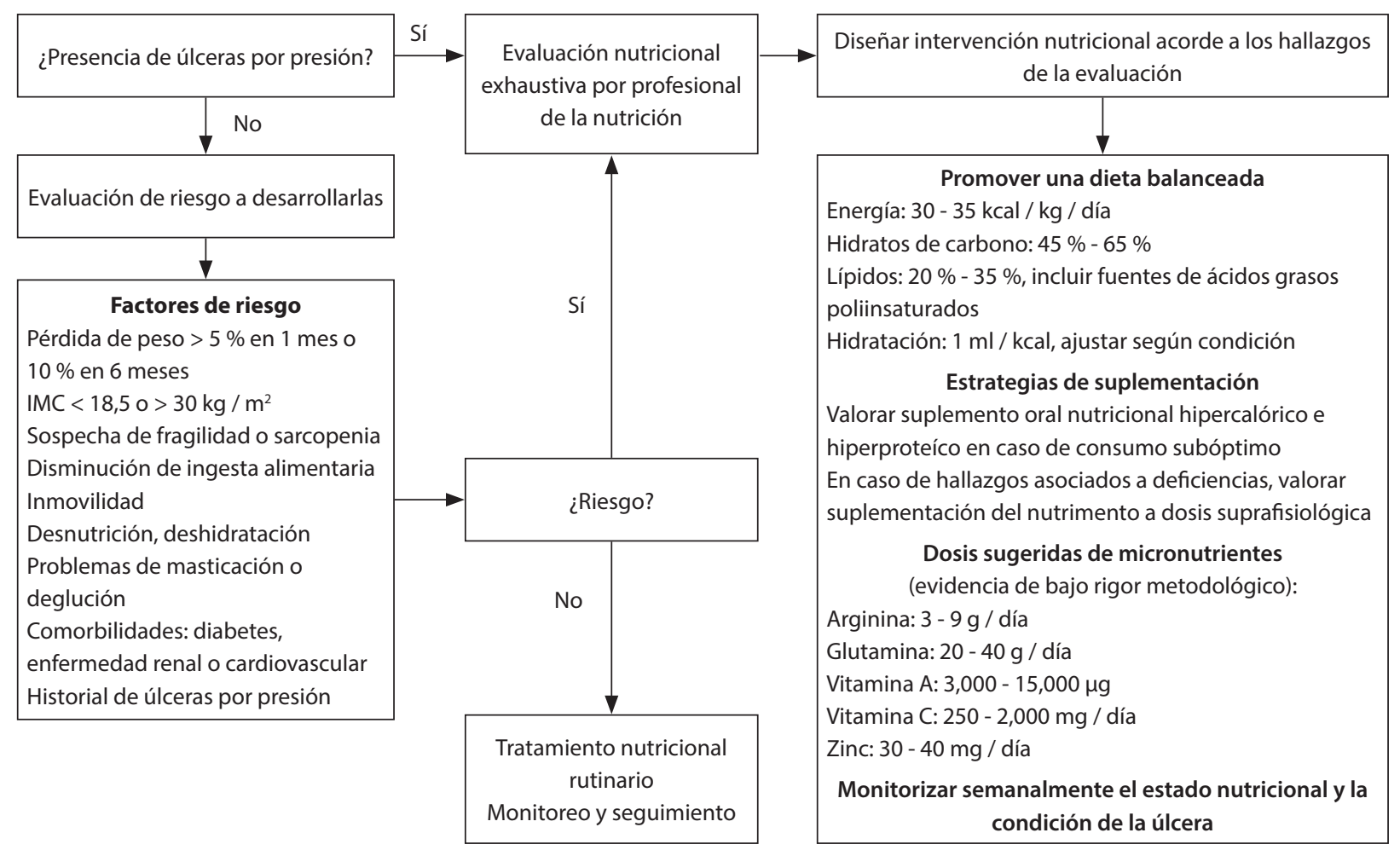

Figura 2. Algoritmo de atención nutricional en el paciente con úlceras por presión. El riesgo de padecer úlceras por presión y la presencia de las mismas, requiere una evaluación nutricional objetiva para poder diseñar una intervención adecuada e individualizada del abordaje nutricional, que incluya el aporte de macro y micronutrientes, líquidos y la valoración del uso de inmunonutrientes. El monitoreo es de suma importancia para valorar la condición de la úlcera.

de hidratación, energía, macronutrientes y micronutrientes clave.

\section{Declaración de autoría}

Todos los autores han participado en la concepción y realización del trabajo que ha dado como resultado el artículo en cuestión. CPMM e IAOP participaron en el análisis de los resultados; PPMV participó en la redacción del trabajo. Todos los autores revisaron el artículo y validaron su versión final.

\section{Declaración de conflictos de interés}

Sin conflictos de interés por declarar.

\section{Financiamiento}

El presente estudio no tuvo financiación.

\section{Referencias bibliográficas}

1. Bernabei R, Manes-Gravina E, Mammarella F. Epidemiologia delle piaghe da cubito. G Gerontol. 2011;59:237-243.

2. Makrantonaki E, Wlaschek M, Scharffetter-Kochanek K. Pathogenesis of wound healing disorders in the elderly. J Dtsch Dermatol Ges. 2017;15(3):255-275. doi: https://doi. org/10.1111/ddg.13199.

3. Khor HM, Tan J, Saedon NI, Kamaruzzaman SB, Chin AV, Poi $\mathrm{PJH}$, et al. Determinants of mortality among older adults with pressure ulcers. Arch Gerontol Geriatr. 2014;59(3):536-41. doi: https://doi.org/10.1016/j.archger.2014.07.011.

4. Zuo X-L, Meng F-J. A care bundle for pressure ulcer treatment in intensive care units. Int J Nurs Sci. 2015;2(4):340-347. doi: https://doi.org/10.1016/j.ijnss.2015.10.008.

5. Shahin ESM, Meijers JMM, Schols JMGA, Tannen A, Halfens RJG, Dassen T. The relationship between malnutrition parameters and pressure ulcers in hospitals and nursing homes. Nutrition. 2010; 26(9): 886-9. doi: https://doi. org/10.1016/j.nut.2010.01.016. 
6. Lorenz HP, Longaker MT. Wounds: Biology, Pathology, and Management. En: Norton JA, Barie PS, Bollinger RR, Chang AE, Lowry SF. Surgery: Basic Science and Clinical Evidence. Springer; 2008:191-208.

7. Avishai E, Yeghiazaryan K, Golubnitschaja O. Impaired wound healing: facts and hypotheses for multi-professional considerations in predictive, preventive and personalised medicine. EPMA J. 2017;8(1):23-33. doi: https://doi.org/10.1007/ s13167-017-0081-y.

8. Frykberg RG, Banks J. Challenges in the Treatment of Chronic Wounds. Adv Wound Care. 2015;4(9):560-82. doi: https:// doi.org/10.1089/wound.2015.0635.

9. Posthauer ME, Banks M, Dorner B, Schols JMGA. The role of nutrition for pressure ulcer management: national pressure ulcer advisory panel, european pressure ulcer advisory panel, and pan pacific pressure injury alliance white paper. Adv Skin Wound Care. 2015;28(4):175-88. doi: https://doi. org/10.1097/01.ASW.0000461911.31139.62.

10. Iizaka S, Okuwa M, Sugama J, Sanada H. The impact of malnutrition and nutrition-related factors on the development and severity of pressure ulcers in older patients receiving home care. Clin Nutr. 2010;29(1):47-53. doi: https://doi. org/10.1016/j.clnu.2009.05.018.

11. Banks MD, Graves N, Bauer JD, Ash S. Cost effectiveness of nutrition support in the prevention of pressure ulcer in hospitals. Eur J Clin Nutr. 2013; 67(1): 42-6. doi: https://doi. org/10.1038/ejcn.2012.140.

12. Haughey L, Barbul A. Nutrition and Lower Extremity Ulcers: Causality and/or Treatment. Int J Low Extrem Wounds. 2017;16(4):238-43. doi: https://doi. org/10.1177/1534734617737639.

13. Neyens JCL, Cereda E, Meijer EP, Lindholm C, Schols JMGA. Arginine-enriched oral nutritional supplementation in the treatment of pressure ulcers: A literature review. Wound Medicine. 2017;16:46-51. doi: https://doi.org/10.1016/j. wndm.2016.07.002.

14. Guo S, DiPietro LA. Factors Affecting Wound Healing. J Dent Res. 2010;89(3):219-29. doi: https://doi. org/10.1177/0022034509359125.

15. Palmieri B, Vadalà M, Laurino C. Nutrition in wound healing: investigation of the molecular mechanisms, a narrative review. J Wound Care. 2019;28(10):683-93. doi: https://doi. org/10.12968/jowc.2019.28.10.683.

16. Litchford MD, Dorner B, Posthauer ME. Malnutrition as a Precursor of Pressure Ulcers. Adv Wound Care. 2014;3(1):5463. doi: https://doi.org/10.1089/wound.2012.0385.

17. Citty SW, Cowan LJ, Wingfield Z, Stechmiller J. Optimizing Nutrition Care for Pressure Injuries in Hospitalized Patients. Ad Wound Care. 2019;8(7):309-22. doi: https://doi. org/10.1089/wound.2018.0925.

18. Jaul E, Barron J, Rosenzweig JP, Menczel J. An overview of co-morbidities and the development of pressure ulcers among older adults. BMC Geriatr. 2018;18(1):305. doi: https://doi. org/10.1186/s12877-018-0997-7.
19. Wilson JA, Clark JJ. Obesity: impediment to postsurgical wound healing. Adv Skin Wound Care. 2004;17(8):426-35. doi: https://doi.org/10.1097/00129334-200410000-00013.

20. Anaya DA, Dellinger EP. The obese surgical patient: a susceptible host for infection. Surg Infect. 2006;7(5):473-80. doi: https://doi.org/10.1089/sur.2006.7.473.

21. Ness SJ, Hickling DF, Bell JJ, Collins PF. The pressures of obesity: The relationship between obesity, malnutrition and pressure injuries in hospital inpatients. Clin Nutr. 2018;37(5):1569-74. doi: https://doi.org/10.1016/j. clnu.2017.08.014.

22. Han G, Ceilley R. Chronic Wound Healing: A Review of Current Management and Treatments. Adv Ther. 2017;34(3):599-610. doi: https://doi.org/10.1007/s12325017-0478-y.

23. Alfonso AR, Kantar RS, Ramly EP, Daar DA, Rifkin W, Levine JP, et al. Diabetes is associated with an increased risk of wound complications and readmission in patients with surgically managed pressure ulcers. Wound Repair Regen. 2019;27(3):249-56. doi: https://doi.org/10.1111/ wrr.12694.

24. Falanga V. Wound healing and its impairment in the diabetic foot. Lancet. 2005;366(9498):1736-43. doi: https://doi. org/10.1016/S0140-6736(05)67700-8.

25. Corniello AL, Moyse T, Bates J, Karafa M, Hollis C, Albert NM. Predictors of pressure ulcer development in patients with vascular disease. J Vasc Nurs. 2014;32(2):55-62. doi: https:// doi.org/10.1016/j.jvn.2013.07.002.

26. Chello C, Lusini M, Schilirò D, Greco SM, Barbato R, Nenna A. Pressure ulcers in cardiac surgery: Few clinical studies, difficult risk assessment, and profound clinical implications. Int Wound J. 2019;16(1):9-12. doi: https://doi.org/10.1111/ iwj.12994.

27. Margolis DJ, Knauss J, Bilker W, Baumgarten M. Medical conditions as risk factors for pressure ulcers in an outpatient setting. Age Ageing. 2003;32(3):259-64. doi: https://doi. org/10.1093/ageing/32.3.259.

28. Munoz N, Posthauer ME, Cereda E, Schols JMGA, Haesler E. The Role of Nutrition for Pressure Injury Prevention and Healing: The 2019 International Clinical Practice Guideline Recommendations. Adv Skin Wound Care. 2020;33(3):123-136. doi: https://doi.org/10.1097/01. ASW.0000653144.90739.ad.

29. Cereda E, Klersy C, Rondanelli M, Caccialanza R. Energy balance in patients with pressure ulcers: a systematic review and meta-analysis of observational studies. J Am Diet Assoc. 2011;111(12):1868-76. doi: https://doi.org/10.1016/j. jada.2011.09.005.

30. U.S. Department of Health and Human Services and U.S. Department of Agriculture. 2015 - 2020 Dietary Guidelines for Americans [Internet]: 8th Edition; December 2015 [Fecha de consulta: Julio 23 de 2020]. Disponible en:https:// health.gov/our-work/food-and-nutrition/2015-2020-dietary-guidelines/. 
31. Theilla M, Schwartz B, Zimra Y, Shapiro H, Anbar R, Rabizadeh E, et al. Enteral n-3 fatty acids and micronutrients enhance percentage of positive neutrophil and lymphocyte adhesion molecules: a potential mediator of pressure ulcer healing in critically ill patients. Br J Nutr. 2012;107(7):10561061. doi: https://doi.org/10.1017/S0007114511004004.

32. Ohura T, Nakajo T, Okada S, Omura K, Adachi K. Evaluation of effects of nutrition intervention on healing of pressure ulcers and nutritional states (randomized controlled trial). Wound Repair Regen. 2011;19(3):330-36. doi: https://doi. org/10.1111/j.1524-475X.2011.00691.x.

33. Dickerson R. Nitrogen Balance and Protein Requirements in Critically ill Older patients. Nutrients. 2016, 8(4): 226. doi: https://doi.org/10.3390/nu8040226.

34. Tiderencel KA, Brody RA. Evidence-Based Interventions for Diabetic Heel Ulcers: Nutrition-Focused Management of a Rehabilitation Patient. Top Clin Nutr. 2017;32(4):305-15. doi: https://doi.org/10.1097/TIN.0000000000000116.

35. Desneves KJ, Todorovic BE, Cassar A, Crowe TC. Treatment with supplementary arginine, vitamin $\mathrm{C}$ and zinc in patients with pressure ulcers: a randomised controlled trial. Clin Nutr. 2005;24(6):979-87. doi: https://doi.org/10.1016/j. clnu.2005.06.011.

36. van Anholt RD, Sobotka L, Meijer EP, Heyman H, Groen HW, Topinková E, et al. Specific nutritional support accelerates pressure ulcer healing and reduces wound care intensity in non-malnourished patients. Nutrition. 2010;26(9):867-72. doi: https://doi.org/10.1016/j.nut.2010.05.009.

37. Collins N. Glutamine and Wound Healing. Adv Skin Wound Care. 2002;15(5):233-34.

38. Blass SC, Goost H, Tolba RH, Stoffel-Wagner B, Kabir K, Burger $\mathrm{C}$, et al. Time to wound closure in trauma patients with disorders in wound healing is shortened by supplements containing antioxidant micronutrients and glutamine: A PRCT. Clin Nutr. 2012;31(4):469-75. doi: https://doi. org/10.1016/j.clnu.2012.01.002.

39. Collins N. Vitamin C and Pressure Ulcers. Adv Skin Wound Care. 2002;15(4):186-88.
40. ter Riet G, Kessels AG, Knipschild PG. Randomized clinical trial of ascorbic acid in the treatment of pressure ulcers. J Clin Epidemiol. 1995;48(12):1453-60. doi: https://doi. org/10.1016/0895-4356(95)00053-4.

41. Livingstone C. Zinc: physiology, deficiency, and parenteral nutrition. Nutr Clin Pract. 2015;30(3):371-82. doi: https:// doi.org/10.1177/0884533615570376.

42. Lin P-H, Sermersheim M, Li H, Lee PHU, Steinberg SM, Ma J. Zinc in Wound Healing Modulation. Nutrients. 2017;10(1):16. doi: https://doi.org/10.3390/nu10010016.

43. Nakamura H, Sekiguchi A, Ogawa Y, Kawamura T, Akai R, Iwawaki $\mathrm{T}$, et al. Zinc deficiency exacerbates pressure ulcers by increasing oxidative stress and ATP in the skin. J Dermatol Sci. 2019;95(2):62-69. doi: https://doi.org/10.1016/j.jdermsci.2019.07.004.

44. Song Y-P, Wang L, Yu H-R, Yuan B-F, Shen H-W, Du L, et al. Zinc Therapy Is a Reasonable Choice for Patients With Pressure Injuries: A Systematic Review and Meta-Analysis. Nutr Clin Pract. 2020. Epub Mar 13. doi: https://doi. org/10.1002/ncp.10485.

45. Langer G, Fink A. Nutritional interventions for preventing and treating pressure ulcers. Cochrane Database Syst Rev. 2014;(6):CD003216. doi: https://doi. org/10.1002/14651858.CD003216.pub2.

46. Cereda E, Neyens JCL, Caccialanza R, Rondanelli M, Schols JMGA. Efficacy of a Disease-Specific Nutritional Support for Pressure Ulcer Healing: A Systematic Review and MetaAnalysis. J Nutr Health Aging. 2017;21(6):655-61. doi: https://doi.org/10.1007/s12603-016-0822-y.

47. Cereda E, Klersy C, Andreola M, Pisati R, Schols JMGA, Caccialanza $\mathrm{R}$, et al. Cost-effectiveness of a disease-specific oral nutritional support for pressure ulcer healing. Clin Nutr. 2017;36(1):246-52. doi: https://doi.org/10.1016/j. clnu.2015.11.012.

48. Wong A, Goh G, Banks MD, Bauer JD. Economic Evaluation of Nutrition Support in the Prevention and Treatment of Pressure Ulcers in Acute and Chronic Care Settings: A Systematic Review. J Parenter Enteral Nutr. 2019;43(3):376400. doi: https://doi.org/10.1002/jpen.1431. 